\title{
Angular Distributions of Electrons Photoemitted from Core Levels of Oriented Diatomic Molecules: Multiple Scattering Theory in Non-Spherical Potentials
}

\author{
R. Díez Muiño ${ }^{1,2}$, D. Rolles ${ }^{2,3}$, F. J. García de Abajo ${ }^{2,4}$, C. S. Fadley ${ }^{2,5}$, and M. A. Van \\ Hove $^{2,5,6}$ \\ ${ }^{1}$ Donostia International Physics Center, P. Manuel de Lardizabal 4, 20018 San Sebastián, Spain, \\ ${ }^{2}$ Materials Sciences Division, Lawrence Berkeley National Laboratory, Berkeley, California \\ 94720, USA \\ ${ }^{3}$ Fritz-Haber-Institut der Max-Planck-Gesellschaft, D-14195 Berlin, Germany \\ ${ }^{4}$ Centro Mixto CSIC-UPV/EHU, Aptdo. 1072, 20080 San Sebastián, Spain \\ ${ }^{5}$ Department of Physics, University of California at Davis, Davis, California 95616, USA \\ ${ }^{6}$ Advanced Light Source, Lawrence Berkeley National Laboratory, Berkeley, California 94720, \\ $U S A$
}

(January 24, 2002)

\begin{abstract}
We use multiple scattering in non-spherical potentials (MSNSP) to calculate the angular distributions of electrons photoemitted from the 1s-shells of $\mathrm{CO}$ and $\mathrm{N}_{2}$ gas-phase molecules with fixed-in-space orientations. For low photoelectron kinetic energies $(E<50 \mathrm{eV})$, as appropriate to certain shaperesonances, the electron scattering must be represented by non-spherical scattering potentials, which are naturally included in our formalism. Our calculations accurately reproduce the experimental angular patterns recently measured by several groups, including those at the shape-resonance energies. The MSNSP theory is thus an accurate and efficient method to calculate the scat-
\end{abstract}


tering states of low-energy electrons in small low-symmetry systems.

PACS numbers: 33.80.Eh,33.90.+h,33.60.Cv

Typeset using REVTEX 
In the past few years, experimental advances have permitted the measurement of the angular distributions of photoelectrons emitted from free molecules fixed in space. Previous to this work, free-molecule studies were limited to orientationally-averaged measurements, thus limiting the information derivable from the data. Most of the experimental work has focused on diatomic molecules [1-7]. The dependence of such fixed-in-space angular distributions on photon energy provides an exciting new probe of electronic structure and dynamics. Particularly important are the energies for which the so-called shape resonances appear in the continuum, since the experimental angular profiles show radical changes. However, the theoretical analysis of the angular patterns has often been limited to the phenomenological fitting of angular distributions to a series of spherical harmonics, with little or no ab-initio input of the relevant parameters $[3,6]$. Quantitative microscopic theories developed some time ago [8,9], while containing good first approximations to the essential physics, have not reproduced accurately the measured angular distributions at the kinetic energies of interest. Further theoretical attempts [10-12] led to the conclusion that more advanced theories were needed to be able to benefit from the information contained in the large amount of experimental data collected in the last few years.

In this Letter, we consider one such advance: the use of multiple scattering of electrons in non-spherical potentials (MSNSP) to calculate the angular distributions of photoelectrons emitted from core levels of small molecules with definite orientations in space. This method is directly derived from approaches previously used in describing photoelectron diffraction from near-surface atoms [13]. Multiple scattering of electrons in space-filling potentials ("full potentials") has a well-founded theoretical basis [14]. However, only very limited use has been made to date of full potentials in photoemission and photoelectron diffraction theory [15], in spite of their being of inherently higher accuracy than the more common sphericalsymmetry muffin-tin approach [16]. Among other benefits, the use of space-filling potentials allows us to overcome one of the limitations of the standard multiple scattering theory between spherical potentials: the approximate treatment of interstitial regions of space in which the potential is neither negligible nor constant (as often assumed). As an example, we 
apply our method to the calculation of the angular distributions of electrons photoemitted from the 1s-shells of $\mathrm{CO}$ and $\mathrm{N}_{2}$. The results are found to be in excellent agreement with recent extensive sets of experimental data [3,4,6,7], and this suggests the general utility of the MSNSP approach for understanding such processes more quantitatively.

From time-dependent first-order perturbation theory, the final state of the photoemitted electron can be obtained from:

$$
\Psi=\left[\hat{\mathrm{G}}^{0}+\hat{\mathrm{G}}^{0} \hat{\mathrm{T}}^{\mathrm{mol}} \hat{\mathrm{G}}^{0}\right] \hat{V}^{\mathrm{rad}} \phi_{i},
$$

where $\phi_{i}$ is the initial state of the electron in the molecule (in the present case, a core state), $\hat{V}^{\mathrm{rad}}$ is the incident-light operator, and $\hat{\mathrm{G}}^{0}$ is the free-electron Green operator. $\hat{\mathrm{T}}^{\mathrm{mol}}$ is the scattering operator of the molecule, that can be obtained in terms of the total molecular potential $\hat{\mathrm{V}}^{\mathrm{mol}}$ in which the photoemitted electron moves. The molecular potential in real space $V^{\mathrm{mol}}(\mathbf{r})$ is calculated as the sum of an electrostatic potential plus a local exchange potential [17]. The electrostatic potential is the Coulomb potential of the nuclei plus the Coulomb potential of the remaining N-1 electrons, the latter calculated from the one-electron Hartree-Fock orbitals, in the frozen-core approximation. $V^{\mathrm{mol}}(\mathbf{r})$ is then split into two different non-spherical cells, within each of which the potential $V^{\alpha}(\mathbf{r})$ is described in a nonspherical form (see the inset of Fig. 1, with $\alpha$ here indexing a given atomic center, $\mathrm{C}$ or $\mathrm{O}$ in the case of $\mathrm{CO}$ ). The size of these cells is limited by the internuclear distance, with no intracell vector being larger than the distance between the two nuclei of the molecule. Outside the cells, the potential is assumed to be zero. Let us remark at this point that the non-sphericity of the potentials inside the cells is due to two features: (a) the actual geometry of the cells, which are non-spherical, and (b) to the potential inside each of the cells, which is the molecular potential of the $\mathrm{CO}^{+}$ion and not any atomic potential.

The restriction in the size of the cells implies that we are neglecting the effect of the Coulomb potential tail at long distances. However, the local effect of the Coulomb hole is explicitly included in the potential. We have performed a number of calculations in model systems to check that neither the Coulomb tail nor the small potential barrier at 
the cell boundaries have significant effects in the angular distribution of the photoelectrons. Inclusion of the Coulomb tail brings just an energy shift in the position of the resonance. Hence the good agreement that we obtain in the position of the resonance is due to the compensation of two effects of opposite sign: the frozen-core approximation (that shifts the resonance to lower energies) and the omission of the Coulomb tail (that shifts it to higher energies).

As a consequence of the partition of the potential, the matrix elements of the total scattering operator of the molecule $T_{L, L^{\prime}}^{\text {mol }}$ in a partial-wave basis set $\left(L, L^{\prime}\right)$ can be calculated as a function of single-cell scattering matrix elements $t_{L, L^{\prime}}^{\alpha}$ [18]. The index $\alpha$ again identifies atom $\alpha$ in the molecule. The calculation of $t_{L, L^{\prime}}^{\alpha}$ requires the numerical solution of the Schrödinger equation, which is transformed into a system of coupled differential equations, for systems without spherical symmetry [19].

The photoemitted electron wavefunction in real space $\Psi(\mathbf{r})$ is now obtained by multiple scattering theory. For each energy, the wavefunction outside the cells is expanded in terms of outgoing Hankel functions $h_{l}^{+}(k r)$ :

$$
\Psi(\mathbf{r})=\sum_{\alpha} \sum_{L} \rho_{L}^{\alpha} h_{l}^{+}\left(k\left|\mathbf{r}-\mathbf{r}_{\alpha}\right|\right) Y_{L}\left(\Omega_{\mathbf{r}-\mathbf{r}_{\alpha}}\right)
$$

where $k$ is the electron momentum $\left(E=k^{2} / 2\right), Y_{L}(\Omega)$ are spherical harmonics and the indices $(l, m)$ are grouped in a single index $L$. The coefficients $\rho_{L}^{\alpha}$ have to be determined. Continuity of the wavefunction at the boundaries of the cells imposes the following multiple scattering conditions [13]:

$$
\rho_{L}^{\alpha}=\left[\rho_{L}^{\alpha}\right]_{0}-i k \sum_{\beta \neq \alpha} \sum_{L_{1}, L_{2}} i^{l-l_{1}} t_{L, L_{1}}^{\alpha} G_{L_{1}, L_{2}}^{\alpha \beta} \rho_{L_{2}}^{\beta}
$$

where the coefficients $\left[\rho_{L}^{\alpha}\right]_{0}$ are those of the wavefunction before the intramolecular scattering takes place, and the matrices $G_{L_{1}, L_{2}}^{\alpha \beta}$ are coefficients in the expansion of Hankel functions about a different center [14]. For diatomic molecules the sum over $\beta$ is limited to a single term. The sums over $L_{1}$ and $L_{2}$ are truncated after convergence with the number of L's has been achieved. In the dipole approximation, the coefficients $\left[\rho_{L}^{\alpha}\right]_{0}$ are calculated from 
the corresponding matrix elements [13]. They depend on the light polarization vector. The multiple scattering system of equations Eq. (3) is solved by matrix inversion in a single self-consistent step, which yields the coefficients $\rho_{L}^{\alpha}$. The latter is equivalent to calculating the multiple scattering series to infinite order, i.e., the wavefunction outside the cells is calculated exactly.

The coefficients $\rho_{L}^{\alpha}$ describe the behavior of the wavefunction at infinity and are sufficient to calculate the photoelectron intensity at the detector position [13]. The wavefunction $\Psi(\mathbf{r})$ of Eq. (2) could be also expanded in terms of Hankel functions centered about a given nuclear position. The coefficients of this expansion would be equivalent to the angular coefficients which are commonly used to fit the experimental angular patterns $[3,6]$. In this respect, our theoretical calculation provides quantitative ab-initio information on these widely used fitting parameters.

One of the most interesting features of the photoemission spectra from molecules is the appearance of shape-resonances in the continuum. Theoretically, the shape resonances are linked to pronounced peaks in the density of states $d n(E) / d E$ induced in the continuum by the molecular potential. $d n(E) / d E$ is calculated by Lloyd's formula [18]:

$$
\frac{d n(E)}{d E}=\frac{2}{\pi} \operatorname{Im} \operatorname{Tr}\left\{\frac{d}{d E}\left[\ln \left(\delta_{L L^{\prime}}-i k T_{L, L^{\prime}}^{\mathrm{mol}}\right)\right]\right\} .
$$

Here, Tr denotes the operation of taking the trace, and Im refers to the imaginary part of a complex number. Notice that Lloyd's formula gives the change in the density of states induced by the presence of the potential, and not the total density of states. The $T_{L, L^{\prime}}^{\mathrm{mol}}$ elements are straigthforwardly calculated in our framework, by using as initial wavefunction before scattering (i.e., the $\left[\rho_{L}^{\alpha}\right]_{0}$ coefficients) the partial wave decomposition of an incident plane wave. The axial symmetry of the molecule keeps the index $m$ as a good quantum number, so that $d n(E) / d E$ can be independently calculated for each value of $m$, but it still involves various l-contributions.

We plot in Fig. 1 the density of states induced in the continuum by a CO molecule with a hole in the $\mathrm{C}$ 1s-shell as a function of the electron energy $E$. The most important $m$ - 
contributions to the total $d n(E) / d E$ are plotted as well. The theoretical calculation shows a clear $\sigma$-shape resonance. The $\sigma$-character of the shape resonance for this photoexcitation process has been experimentally observed by comparison of the total photoemission cross section for parallel and perpendicular polarizations of the incident light, as measured with respect to the molecular axis [20]. Notice, however, that the $\sigma$-contribution to $d n(E) / d E$ is built by summing over various l-wave components. In the dipole approximation, the only component that will contribute to the photoemission cross-section from the $\mathrm{C}$ 1s orbital would be the one with $p$-character at the origin.

As an example for the power of our method, we show in Fig. 2 the angular distribution of electrons photoemitted from the 1 s-shell of $\mathrm{C}$ in the $\mathrm{CO}$ molecule, when the incident light is linearly polarized and the polarization vector $\varepsilon$ is parallel to the molecular axis. The experimental resolution in energy and angle are included in our theoretical calculation. Two different kinetic energies of the electron are considered: one at the shape resonance $(E=10.4 \mathrm{eV})$, and a second one well above it $(E=21.0 \mathrm{eV})$. The theoretical calculations agree very well with recent experimental data $[3,4,7]$. For kinetic energies above the shape resonance, the electron intensity along the $\mathrm{C}$ direction is higher than the electron intensity along the $\mathrm{O}$ direction [2]. This behaviour is reversed at the shape resonance. The shape resonance thus implies special conditions of scattering for which the directly emitted wave and the scattered waves combine to create constructive interference along the $\mathrm{O}$ direction.

When the diatomic molecule is homonuclear, the theoretical description of the photoemission process is more complex, because of the higher symmetry and the resulting small energy differences between different initial core states [21]. In the case of $\mathrm{N}_{2}$, there are two different energy levels for the 1s-shell electrons: a symmetrical gerade state (with "bonding" character, whose wavefunction can be approximately described by $\Phi_{g} \propto \phi_{1 s}^{a}+\phi_{1 s}^{b}$, with $\phi_{1 s}$ the wavefunction of the 1s-level in atomic N, and $a$ and $b$ the two N atoms involved), and an antisymmetrical ungerade state (with "antibonding" character, and $\Phi_{u} \propto \phi_{1 s}^{a}-\phi_{1 s}^{b}$ ). The energy difference between these two levels is only $\approx 97 \mathrm{meV}[21]$. As a consequence, experimental spectra usually measure the (incoherent) sum of intensities of photoelectrons emitted 
from both states. Our theoretical calculations can however fully allow for this difference in the two initial states, as might be measured in some future experiments at higher energy resolution.

We show in Fig. 3 the angular distribution of electrons photoemitted from the 1s-shell of $\mathrm{N}_{2}$. We consider photoelectrons with kinetic energy at the shape resonance $(E=10 \mathrm{eV}$ in this case). The 1s-shell hole generated by the photoelectron excitation is considered as delocalized in our calculation (i.e., distributed over the two atoms of the molecule). Two different theoretical calculations are plotted in Fig. 3: the photoemission pattern from the 1sderived gerade state and the photoemission pattern from the ungerade state. The incoherent sum of both patterns, as appropriate to independent emission from both types of states, is shown in the figure as well. The summed intensity is in excellent agreement with the experimental data of Weber et al. [7], confirming that the experimental spectra includes contributions from both initial states, of necessity because the experimental energy window is larger than the level splitting.

In summary, we have shown that multiple scattering in non-spherical potentials (MSNSP) is a powerful theoretical method for studying photoelectron angular distributions from molecules oriented in space. It very accurately describes the photoemission angular distributions of gas-phase oriented diatomic molecules, like $\mathrm{CO}$ and $\mathrm{N}_{2}$, including energies close to the shape resonance, a severe case for which the angular distributions are extremely sensitive to the details of the calculation. The expansion of the wavefunction in partial waves used in our formalism provides all information necessary for an analysis of a complete photoionization experiment with the available experimental data. Beyond the calculations described here, other topics of high current interest to which MSNSP should be applicable are non-dipole and circular dichroism effects in photoemission from oriented molecules.

Furthermore, MSNSP has potential applicability in many other spectroscopies, such as photoemission, photoelectron diffraction, NEXAFS, or ion-induced electron emission. The computational efficiency of other ab-initio methods in the calculation of low-energy oneelectron excited states is much reduced in low-symmetry systems (adsorbates, clusters, and 
nanostructures). Our results on small molecules show that MSNSP is a fast and accurate alternative method to calculate the low-energy scattering states of electrons in such systems.

\section{ACKNOWLEDGEMENTS}

This work was supported in part by the Director, Office of Science, Office of Basic Energy Sciences, Division of Materials Sciences of the U.S. Department of Energy under Contract No. DE-AC03-76SF00098. R. D. M. acknowledges financial support by the Gipuzkoako Foru Aldundia (Gipuzkoa Fellows Program). 


\section{REFERENCES}

[1] E. Shigemasa, J. Adachi, M. Oura, and A. Yagishita, Phys. Rev. Lett. 74, 359 (1995).

[2] F. Heiser, O. Gessner, J. Viefhaus, K. Wieliczek, R. Hentges, and U. Becker, Phys. Rev. Lett. 79, 2435 (1997).

[3] E. Shigemasa, J. Adachi, K. Soejima, N. Watanabe, A. Yagishita, and N. A. Cherepkov, Phys. Rev. Lett. 80, 1622 (1998).

[4] S. Motoki, J. Adachi, Y. Hikosaka, K. Ito, M. Sano, K. Soejima, A. Yagishita, G. Raseev, and N. A. Cherepkov, J. Phys. B 33, 4193 (2000).

[5] A. Lafosse, M. Lebech, J. C. Brenot, P. M. Guyon, O. Jagutzki, L. Spielberger, M. Vervloet, J. C. Houver, and D. Dowek, Phys. Rev. Lett. 84, 5987 (2000).

[6] A. Landers, Th. Weber, I. Ali, A. Cassimi, M. Hattass, O. Jagutzki, A. Nauert, T. Osipov, A. Staudte, M. H. Prior, H. Schmidt-Böcking, C. L. Cocke, and R. Dörner, Phys. Rev. Lett. 87, 013002 (2001).

[7] T. Weber et al., J. Phys. B (in press).

[8] D. Dill and J. L. Dehmer, J. Chem. Phys. 61, 692 (1974).

[9] D. Dill, J. Siegel, and J. L. Dehmer, J. Chem. Phys. 65, 3158 (1976).

[10] K. Ito, J. Adachi, Y. Hikosaka, S. Motoki, K. Soejima, A. Yagishita, G. Raseev, and N. A. Cherepkov, Phys. Rev. Lett. 85, 46 (2000).

[11] N. A. Cherepkov, S. K. Semenov, Y. Hikosaka, K. Ito, S. Motoki, and A. Yagishita, Phys. Rev. Lett. 84, 250 (2000).

[12] N. A. Cherepkov, G. Raseev, J. Adachi, Y. Hikosaka, K. Ito, S. Motoki, M. Sano, K. Soejima, and A. Yagishita, J. of Phys. B 33, 4213 (2000).

[13] F. J. García de Abajo, M. A. Van Hove, and C. S. Fadley, Phys. Rev. B 63, 075404 
(2001); see also http://electron.lbl.gov/ edac.

[14] A. Gonis and W. H. Butler, Multiple Scattering in Solids, (Springer-Verlag Inc., New York, 2000).

[15] M. Grass, J. Braun, and G. Borstel, Phys. Rev. B 5014827 (1994).

[16] R. Díez Muiño, D. Rolles, F. J. García de Abajo, F. Starrost, W. Schattke, C. S. Fadley, and M. A. Van Hove, J. of Electr. Spectr. and Rel. Phen. 114-116, 99 (2001).

[17] J. Siegel, J. L. Dehmer, and D. Dill, Phys. Rev. A 21, 85 (1980).

[18] P. Lloyd and P. V. Smith, Adv. Phys. 21, 69 (1972).

[19] S. Nagano and S. Y. Tong, Phys. Rev. B 32, 6562 (1985).

[20] E. Shigemasa, T. Hayaishi, T. Sasaki, and A. Yagishita, Phys. Rev. A 47, 1824 (1993).

[21] U. Hergenhahn, O. Kugeler, A. Rüdel, E. E. Rennie, and A. M. Bradshaw, J. Phys. Chem. A 105, 5704 (2001). 
FIGURES

FIG. 1. Theoretical calculation of the density of states in the continuum $d n(E) / d E$ (in units of $\mathrm{eV}^{-1}$ ), as a function of the kinetic energy of the electron $(\mathrm{eV})$ for a $\mathrm{CO}$ molecule with a hole in the 1s-shell of the $\mathrm{C}$ atom. The solid line is the total density of states. The dashed, dash-dotted and dotted line respectively are the $\sigma(m=0), \pi(|m|=1)$, and $\delta(|m|=2)$ contributions to the total density of states. A schematic of the potential partition is shown in the inset.

FIG. 2. Theoretical calculation of the angular distribution of electrons photoemitted from the 1s-shell of $\mathrm{C}$ in $\mathrm{CO}$, with light polarization $\varepsilon$ along the $\mathrm{C}-\mathrm{O}$ axis (solid line). The $\mathrm{O}$ atom is at 0 degrees (right side of the image), and the $\mathrm{C}$ atom is at 180 degrees (left side of the image). The kinetic energy of the electrons is $10.4 \mathrm{eV}$ in panel (a) and $21.0 \mathrm{eV}$ in panel (b). The black circles, squares and triangles respectively are experimental data from Ref. [3], Ref. [4], and Ref. [7]. Arbitrary intensity units are used.

FIG. 3. Theoretical calculation of the angular distribution of electrons photoemitted from the 1s-derived levels of $\mathrm{N}_{2}$, with light polarization $\varepsilon$ along the $\mathrm{N}-\mathrm{N}$ axis. The kinetic energy of the electrons is $10.0 \mathrm{eV}$. The thin dashed line is the angular distribution of electrons photoemitted from the gerade core state. The thin solid line is the angular distribution of electrons photoemitted from the ungerade core state. The thick solid line is the sum of both contributions. The black squares are experimental data [7]. Arbitrary intensity units are used. 